EPJ manuscript No.

(will be inserted by the editor)

\title{
Scalar Mesons in a Chiral Quark Model with Glueball.
}

\author{
D. Ebert ${ }^{1}$, M. Nagy ${ }^{2}$, M. K. Volkov ${ }^{3}$, and V. L. Yudichev ${ }^{3}$ \\ 1 Institut für Physik, Humboldt-Universität zu Berlin, Invalidenstrasse 110, D-10115 Berlin, Germany \\ 2 Institute of Physics, Slovak Academy of Sciences, 84228 Bratislava, Slovakia \\ 3 Bogoliubov Laboratory of Theoretical Physics, Joint Institute for Nuclear Research,141980 Dubna, Russia
}

the date of receipt and acceptance should be inserted later

\begin{abstract}
Ground-state scalar isoscalar mesons and a scalar glueball are described in a $U(3) \times U(3)$ chiral quark model of the Nambu-Jona-Lasinio (NJL) type with 't Hooft interaction. The latter interaction produces singlet-octet mixing in the scalar and pseudoscalar sectors. The glueball is introduced into the effective meson Lagrangian as a dilaton on the base of scale invariance. The mixing of the glueball with scalar isoscalar quarkonia and amplitudes of their decays into two pseudoscalar mesons are shown to be proportional to current quark masses, vanishing in the chiral limit. Mass spectra of the scalar mesons and the glueball and their main modes of strong decay are described.
\end{abstract}

PACS. 12.39.Ki Relativistic quark model - 12.39.Mk Glueball and nonstandard multiquark/gluon states - 13.25.-k Hadronic decays of mesons - 14.40.-n Mesons

\section{Introduction}

Scalar mesons play an important role in the strong interactions of elementary particles [1,2]. They are also significant for a correct description of nuclear interactions [3]. Today, 19 scalar resonances are observed in the energy interval from 0.4 to $1.7 \mathrm{GeV}$ [1]. However, their nature is not yet quite clear. Are they two-quark or four-quark states? Which of them belong to the ground state nonet of the $U(3)$ flavour group; and which, to the excited one? Is there a glueball among them, and where does it lie? These are intriguing questions, where to find answers, many attempts are currently being made by different authors. From a quick survey of what has been done by now, one could conclude that we do not yet approach the solution. A lot of models, (see, e.g., 15, 6, 7, 8, 9, 10, 11, 12, 13, 14, 15, 16, 17, $18,19,20,21,22$ ) has been suggested, but none of the approaches has given us the clue that could allow us to clarify the true nature of scalar mesons once for all. Moreover, one might be disappointed by the fact that different models give different results that do not much overlap, being in some cases controversial.

In our work, we describe scalar isoscalar mesons and their mixing with scalar glueballs. As we learn from QCD, if there were no quarks in the world, gluons themselves could form bound objects due to a large coupling strength of gluon self-interactions. This stimulated the search for bound gluon systems both in experiment and in theory. As the gluon carries no flavour, lepton or baryon quantum number, glueballs must be searched among isoscalar mesons. Indeed, the simplest gluonic formation possesses

Correspondence to: volkov@thsun1.jinr.ru the quantum numbers of a scalar isoscalar meson. If one looks into tables of experimental data, one finds a large number of scalar isoscalar states that can be mixed states of quarkonia, multi-quark systems, hybrids, and glueballs. To distinguish a glueball is really a difficult task, because we have no reliable test that would give us the truth.

As the perturbative approach does not work here, different phenomenological models and lattice simulations are involved in the study. From recent results [23, 24, 25] one can conclude that it is most probably that glueballs are real objects of our world. There exist numerical estimates for probable masses of glueballs, however still in the world without quarks. Lattice calculations report that the lightest scalar glueball should be found between 1.5 and $1.7 \mathrm{GeV}$.

Amsler 18 considered the state $f_{0}(1500)$ as a candidate for the scalar glueball. QCD sum rules 19 and the K-matrix method [20] showed that both $f_{0}(1500)$ and $f_{0}(1710)$ are mixed states with large admixture of the glueball component. Moreover, QCD sum rules [19] require that light glueballs (below $1 \mathrm{GeV}$ ) should exist, which is in contradiction with what lattice calculations suggest.

A glueball cannot be searched without investigating the nature of the rest of scalar mesons that are not heavier than, $2 \mathrm{GeV}$ and which we consider mostly as formed by quark-antiquark pairs. All the bound isoscalar $q \bar{q}$ states are allowed to mix with glueballs, and their spectrum has many interpretations made by different authors. For instance, Palano [26] suggested a scenario, in which the states $a_{0}(980), K_{0}^{*}(1430), f_{0}(980)$, and $f_{0}(1400)$ form a nonet. The state $f_{0}(1500)$ is considered as the scalar glue- 
D. Ebert, M. Nagy, M. K. Volkov,, V. L. Yudichev: Scalar Mesons in a Chiral Quark Model with Glueball.

ball. Törnqvist et al. 21 looked upon the states $f_{0}(980)$ and $f_{0}(1370)$ as manifestations of the ground and excited $s \bar{s}$ states; and the state $f_{0}(400-1200)$, as the ground $u \bar{u}$ state. Van Beveren et al. 27 considered the states $f_{0}(400-1200)$ and $f_{0}(1370)$ as $u \bar{u}$ ground states; and the states $f_{0}(980)$ and $f_{0}(1500)$, as $s \bar{s}$ ground states. Two ground states for each $q \bar{q}$ system occur due to pole doubling, which takes place for scalar mesons in their model. Shakin et al. 15 obtained from a nonlocal confinement model that the $f_{0}(980)$ resonance is the ground $u \bar{u}$ state, and $f_{0}(1370)$ is the ground $s \bar{s}$ state. The state $f_{0}(1500)$ is considered as a radial excitation of $f_{0}(980)$. They believed the mass of the scalar glueball to be $1770 \mathrm{MeV}$.

In our recent papers [17, following the methods given in Refs. 22,28,29, 30, we showed that all experimentally observed scalar meson states with masses in the interval from 0.4 to $1.71 \mathrm{GeV}$ can be interpreted as members of two scalar meson nonets - the ground state of the meson-nonet and its first radial excitation. We considered all scalar mesons as $q \bar{q}$ states and took into account the singlet-octet mixing caused by the 't Hooft interaction. In [17], we obtained a scalar isoscalar state with mass 1600 $\mathrm{MeV}$ and had to choose, to which of the experimentally observed states $f_{0}(1500)$ and $f_{0}(1710)$ we should ascribe it. From our analysis of the decay rates calculated in our model, we found that $f_{0}(1710)$ better fits to the nonet of quarkonia than $f_{0}(1500)$. Therefore, we supposed that the state $f_{0}(1500)$ contained a significant component of the scalar glueball (see [19,20]). However, the final decision should be made after including the scalar glueball into the model, and taking account of its mixing with quarkonia, which will shift scalar meson masses.

At present, there exist two candidates for the glueball: $f_{0}(1500)$ and $f_{0}(1710)$ 20,21,22. To describe the properties and mixing of the glueball with the other scalar states, one should introduce an additional scalar isoscalar dilaton field $\chi$ into our model, in addition to the quarkonia which have already been described 17. For this purpose, one can make use of the idea of approximate scale invariance of effective Lagrangians based on the dilaton model. Such models were studied by many authors (see, e. g., 10,22, 31, 32, 33). Unfortunately, there is no unique way to introduce the dilaton field into a chiral Lagrangian. This justifies the large number of models dealing with glueballs.

The guideline, one should follow when introducing the dilaton field into an effective meson Lagrangian, is to reproduce the Ward identity connected with the scale anomaly. The latter leads to the following equation for the vacuum expectation value of the divergence of the dilatation current

$$
\begin{gathered}
\left\langle\partial_{\mu} S^{\mu}\right\rangle=\mathcal{C}_{g}-\sum_{q=u, d, s} m_{q}^{0}\langle\bar{q} q\rangle, \\
\mathcal{C}_{g}=\left(\frac{11}{24} N_{c}-\frac{1}{12} N_{f}\right)\left\langle\frac{\alpha}{\pi} G_{\mu \nu}^{2}\right\rangle,
\end{gathered}
$$

where $N_{c}$ is the number of colours; $N_{f}$, the number of flavours; $\left\langle\frac{\alpha}{\pi} G_{\mu \nu}^{2}\right\rangle$ and $\langle\bar{q} q\rangle$, the gluon and quark condensates; $m_{q}^{0}$, the current quark mass.

In this paper, we are going to use the most natural method of introducing the dilaton field into the effective
Lagrangian by requiring that, in the chiral limit, our Lagrangian should be scale-invariant except for the dilaton potential. To realize this program, one should multiply all dimensional parameters of the original Lagrangian (without dilaton) by a corresponding power of the dilaton field divided by its vacuum expectation value $\chi_{c}$ to preserve the dimensions of model parameters. Thus, instead of the four-quark coupling constant $G$, the 't Hooft coupling constant $K$, ultraviolet cutoff $\Lambda$ (necessary for the regularization of divergent integrals coming from quark loops), and the constituent quark masses $m_{q}(q=u, s)$, one should use $G\left(\chi_{c} / \chi\right)^{2}, K\left(\chi_{c} / \chi\right)^{5}, \Lambda\left(\chi / \chi_{c}\right)$ and $m_{q}\left(\chi / \chi_{c}\right)$.

Current quark masses $m_{q}^{0}$ are not multiplied by the dilaton field and violate scale invariance explicitly, as it takes place in QCD. Their contribution to the divergence of the dilatation current is determined by quark condensates and disappears in the chiral limit (see (1) ).

Omitting, for a moment, the 't Hooft interaction in our approach (which, to an extent, is in the spirit of papers 10 , 22,29 ), we require that the Lagrangian is scale-invariant in the chiral limit both before and after the spontaneous breaking of chiral symmetry (SBCS), except for the dilaton potential. This property can be obtained by considering (after bosonization when the effective Lagrangian is expressed in terms of bosonic scalar and pseudoscalar fields $\sigma$ and $\phi$ ) the shift of the scalar meson field $\sigma$

$$
\sigma=\sigma^{\prime}-m \frac{\chi}{\chi_{c}}, \quad\left(m^{0}=0\right),
$$

where $\left\langle\sigma^{\prime}\right\rangle_{0}=0, \quad\langle\sigma\rangle_{0}=-m$, guaranteing that the relation (11) is satisfied 38. The nonzero vacuum expectation value of $\sigma$ appears as a result of SBCS, and thus, the constituent quark mass is produced. In the case of nonvanishing current quark masses, (3) changes by including an additional (non-scaled) mass term $m^{0}$ into the r.h.s. This change produces an interaction term $\sim \frac{m^{0}}{G}\left(\frac{\chi}{\chi_{c}}\right)^{2} \sigma^{\prime}$ in the effective Lagrangian (31) which breaks both chiral and scale symmetry just in the way required by the quark mass term $m^{0} \bar{q} q$ of the QCD Lagrangian.

The structure of the paper is as follows. In Section 2, we derive the usual $U(3) \times U(3)$-flavour symmetric effective Lagrangian with the 't Hooft interaction and without dilaton fields. In Section 3, the dilaton field is introduced into the effective Lagrangian obtained in Section 2. Gap . equations are investigated in Section 4. In Section 5, we derive mass terms and fix the model parameters. The main decays of scalar isoscalar mesons are calculated in Section 6. Finally, in the Conclusion, we discuss the obtained results.

\section{Chiral effective Lagrangian with 't Hooft interaction}

A $U(3) \times U(3)$ chiral Lagrangian with the 't Hooft interaction was investigated in paper 34]. It consists of three terms as shown in formula (4). The first term represents 
the free quark Lagrangian, the second is composed of fourquark vertices as in the NJL model, and the last one describes the six-quark 't Hooft interaction 35] that is necessary to solve the $U_{A}(1)$ problem.

$$
\begin{aligned}
L= & \bar{q}\left(i \hat{\partial}-m^{0}\right) q+\frac{G}{2} \sum_{a=0}^{8}\left[\left(\bar{q} \lambda_{a} q\right)^{2}+\left(\bar{q} i \gamma_{5} \lambda_{a} q\right)^{2}\right]- \\
& -K\left\{\operatorname{det}\left[\bar{q}\left(1+\gamma_{5}\right) q\right]+\operatorname{det}\left[\bar{q}\left(1-\gamma_{5}\right) q\right]\right\} .
\end{aligned}
$$

Here $G$ and $K$ are coupling constants, $\lambda_{a}(a=1, \ldots, 8)$ are the Gell-Mann matrices $\lambda_{0}=\sqrt{2 / 3} \mathbf{1}$, with 1 being the unit matrix; $m^{0}$ is a current quark mass matrix with diagonal elements $m_{u}^{0}, m_{d}^{0}, m_{s}^{0}\left(m_{u}^{0} \approx m_{d}^{0}\right)$.

The standard bosonization procedure for local quark models consists in replacing the four-quark vertices by Yukawa couplings of quarks with bosonic fields which enables one to perform the integration over quark fields. The final effective bosonic Lagrangian appears then as a result of the calculation of the quark determinant. To realize this program, it is necessary, using the method described in 34, 35, 36, 37, to go from Lagrangian (4) to an intermediate Lagrangian which contains only four-quark vertices

$$
\begin{aligned}
L & =\bar{q}\left(i \hat{\partial}-\bar{m}^{0}\right) q+\frac{1}{2} \sum_{a, b=1}^{9}\left[G_{a b}^{(-)}\left(\bar{q} \tau_{a} q\right)\left(\bar{q} \tau_{b} q\right)\right. \\
& \left.+G_{a b}^{(+)}\left(\bar{q} i \gamma_{5} \tau_{a} q\right)\left(\bar{q} i \gamma_{5} \tau_{b} q\right)\right]
\end{aligned}
$$

where

$$
\begin{aligned}
& \tau_{a}=\lambda_{a} \quad(a=1, \ldots, 7), \quad \tau_{8}=\left(\sqrt{2} \lambda_{0}+\lambda_{8}\right) / \sqrt{3} \\
& \tau_{9}=\left(-\lambda_{0}+\sqrt{2} \lambda_{8}\right) / \sqrt{3} \\
& G_{11}^{( \pm)}=G_{22}^{( \pm)}=G_{33}^{( \pm)}=G \pm 4 K m_{s} I_{1}^{\Lambda}\left(m_{s}\right) \\
& G_{44}^{( \pm)}=G_{55}^{( \pm)}=G_{66}^{( \pm)}=G_{77}^{( \pm)}=G \pm 4 K m_{u} I_{1}^{\Lambda}\left(m_{u}\right) \\
& G_{88}^{( \pm)}=G \mp 4 K m_{s} I_{1}^{\Lambda}\left(m_{s}\right), \quad G_{99}^{( \pm)}=G \\
& G_{89}^{( \pm)}=G_{98}^{( \pm)}= \pm 4 \sqrt{2} K m_{u} I_{1}^{\Lambda}\left(m_{u}\right) \\
& G_{a b}^{( \pm)}=0 \quad(a \neq b ; \quad a, b=1, \ldots, 7) \\
& \quad m_{u}^{0}=m_{u}^{0}-32 K m_{u} m_{s} I_{1}^{\Lambda}\left(m_{u}\right) I_{1}^{\Lambda}\left(m_{s}\right) \\
& \quad m_{s}^{0}=m_{s}^{0}-32 K m_{u}^{2} I_{1}^{\Lambda}\left(m_{u}\right)^{2}
\end{aligned}
$$

Here $m_{u}$ and $m_{s}$ are constituent quark masses and the integrals

$$
I_{n}^{\Lambda}\left(m_{a}\right)=\frac{N_{c}}{(2 \pi)^{4}} \int d_{e}^{4} k \frac{\theta\left(\Lambda^{2}-k^{2}\right)}{\left(k^{2}+m_{a}^{2}\right)^{n}}, \quad(n=1,2 ; a=u, s),
$$

are calculated in the Euclidean metric and regularized by a simple $O(4)$-symmetric ultraviolet cutoff $\Lambda$. For $I_{1}^{\Lambda}\left(m_{a}\right)$ one gets

$$
I_{1}^{\Lambda}\left(m_{a}\right)=\frac{N_{c}}{16 \pi^{2}}\left(\Lambda^{2}-m_{a}^{2} \ln \left(\frac{\Lambda^{2}}{m_{a}^{2}}+1\right)\right),
$$

where $m_{a}$ represents a corresponding constituent quark mass $^{1}: m_{u}$ or $m_{s}$. Note that we have introduced the notation of constituent quark mass already here, although they will be consistently considered only later, when discussing mass gap equations (compare (41) and (42)) and the related shift of scalar meson fields (see (12) and (13)). However, as we want to use an effective four-fermion interaction instead of the original six-quark one, we have to calculate quark loop corrections for the constant $G$ (see (6)) using full quark propagators with constituent quark masses.

In addition to the one-loop corrections to the constant $G$ at four-quark vertices, we have to modify the current quark masses $m_{a}^{0}$ (see (7) and (8)). This is to avoid the problem of double counting of the 't Hoot contribution in gap equations which was encountered by the author in [37. After the redefinition of the constant $G$ and of the current quark masses, we can guarantee that in the large- $N_{c}$ limit the mass spectrum of mesons and the gap equations, derived from the new Lagrangian with modified four-quark vertices, are the same as those which are obtained from the original Lagrangian with six-quark vertices.

Now we can bosonize Lagrangian (5). By introducing auxiliary scalar $\sigma$ and pseudoscalar $\phi$ fields, we obtain [2, 28,34

$$
\begin{aligned}
\mathcal{L}(\sigma, \phi)= & -\frac{1}{2} \sum_{a, b=1}^{9}\left(\sigma_{a}\left(G^{(-)}\right)_{a b}^{-1} \sigma_{b}+\phi_{a}\left(G^{(+)}\right)_{a b}^{-1} \phi_{b}\right) \\
& -i \operatorname{Tr} \ln \left\{i \hat{\partial}-\bar{m}^{0}+\sum_{a=1}^{9} \tau_{a}\left(\sigma_{a}+i \gamma_{5} \phi_{a}\right)\right\}
\end{aligned}
$$

As we expect, the chiral symmetry is spontaneously broken due to the strong attraction of quarks in the scalar channel and the scalar isoscalar fields acquire nonzero vacuum expectation values $\left\langle\sigma_{a}\right\rangle_{0} \neq 0(a=8,9)$. These values are related to basic model parameters $G, m^{0}$ and $\Lambda$ via gap equations as it will be shown in the next Section. Therefore, we first have to shift the $\sigma$ fields by proper values so that the new fields have zero vacuum expectation values

$$
\sigma_{a}=\sigma_{a}^{\prime}-\mu_{a}+\bar{\mu}_{a}^{0}, \quad\left\langle\sigma_{a}^{\prime}\right\rangle_{0}=0
$$

where $\mu_{a}=0, \quad(a=1, \ldots, 7), \mu_{8}=m_{u}, \mu_{9}=-m_{s} / \sqrt{2}$ and $\bar{\mu}_{a}^{0}=0, \quad(a=1, \ldots, 7), \bar{\mu}_{8}^{0}=\bar{m}_{u}^{0}, \bar{\mu}_{9}^{0}=-\bar{m}_{s}^{0} / \sqrt{2}$. After this shift we obtain:

$$
\begin{gathered}
\mathcal{L}\left(\sigma^{\prime}, \phi\right)=L_{G}\left(\sigma^{\prime}, \phi\right)-i \operatorname{Tr} \ln \{i \hat{\partial}-m \\
\left.+\sum_{a=1}^{9} \tau_{a}\left(\sigma_{a}^{\prime}+i \gamma_{5} \phi_{a}\right)\right\}
\end{gathered}
$$

where

$$
L_{G}\left(\sigma^{\prime}, \phi\right)=
$$

1 The notation "constituent" quark mass refers here to the total quark mass appearing in the full quark propagator. 


$$
\begin{aligned}
& -\frac{1}{2} \sum_{a, b=1}^{9}\left(\sigma_{a}^{\prime}-\mu_{a}+\mu_{a}^{0}\right)\left(G^{(-)}\right)_{a b}^{-1}\left(\sigma_{b}^{\prime}-\mu_{a}+\mu_{a}^{0}\right) \\
& -\frac{1}{2} \sum_{a, b=1}^{9} \phi_{a}\left(G^{(+)}\right)_{a b}^{-1} \phi_{b} .
\end{aligned}
$$

From Lagrangian (13) we take only those terms (in momentum space) which are linear, squared, cubic and quartic in scalar and pseudoscalar fields.2

$$
\begin{aligned}
& \mathcal{L}\left(\sigma^{\prime}, \phi\right)=L_{G}\left(\sigma^{\prime}, \phi\right)+\operatorname{tr}\left[I_{2}^{\Lambda}(m)\left(\sigma^{\prime 2}+\phi^{2}\right)\right. \\
& -4 m I_{1}^{\Lambda}(m) \sigma^{\prime}+2 I_{1}^{\Lambda}(m)\left(\sigma^{\prime 2}+\phi^{2}\right) \\
& -4 m^{2} I_{2}^{\Lambda}(m) \sigma^{\prime 2}+4 m I_{2}^{\Lambda}(m) \sigma^{\prime}\left(\sigma^{\prime 2}+\phi^{2}\right)^{2} \\
& -I_{2}^{\Lambda}(m)\left(\sigma^{\prime 2}+\phi^{2}\right)^{2}+I_{2}^{\Lambda}(m)\left[\sigma^{\prime}-m, \phi\right]_{-}^{2}, \\
& \sigma^{\prime}=\sum_{a=1}^{9} \sigma_{a} \tau_{a}, \quad \phi=\sum_{a=1}^{9} \phi_{a} \tau_{a},
\end{aligned}
$$

where "tr" means calculating the trace over $\tau$-matrix expressions and $[\ldots]_{-}$stands for a commutator [2]. The expression for $I_{1}^{\Lambda}\left(m_{a}\right)$ in Euclidean metric is given in (10). The integrals $I_{2}^{\Lambda}\left(m_{a}\right)$ are also calculated in Euclidean spacetime

$$
I_{2}^{\Lambda}\left(m_{a}\right)=\frac{N_{c}}{16 \pi^{2}}\left(\ln \left(\frac{\Lambda^{2}}{m_{a}^{2}}+1\right)-\frac{\Lambda^{2}}{\Lambda^{2}+m_{a}^{2}}\right) .
$$

Then, we renormalize the fields in (15) so that the kinetic terms of the effective Lagrangian are of conventional form, and diagonalize the isoscalar sector.

$$
\begin{aligned}
\overline{\mathcal{L}}\left(\sigma^{r}, \phi^{r}\right)=\bar{L}_{G}\left(\sigma^{r}, \phi^{r}\right) \\
+\operatorname{tr}\left[\frac{p^{2}}{4}\left(\sigma^{r 2}+\phi^{r 2}\right)-4 m g I_{1}^{\Lambda}(m) \sigma^{r}\right. \\
+2 g^{2} I_{1}^{\Lambda}(m)\left(\sigma^{r 2}+Z \phi^{r 2}\right)+\frac{1}{4}\left[m, \phi^{r}\right]_{-}^{2} \\
-m^{2} \sigma^{r 2}+m g \sigma^{r}\left(\sigma^{r 2}+Z \phi^{r 2}\right)-\frac{1}{2}\left[m, \phi^{r}\right]_{-}\left[\sigma^{r}, \phi^{r}\right]_{-} \\
\left.-\frac{g^{2}}{4}\left(\left(\sigma^{r 2}+Z \phi^{r 2}\right)^{2}-\left[\sigma^{r}, \phi^{r}\right]_{-}^{2}\right)\right], \\
\sigma^{r}=\sum_{a=1}^{9} \sigma_{a}^{r} \tau_{a}, \quad \phi^{r}=\sum_{a=1}^{9} \phi_{a}^{r} \tau_{a} .
\end{aligned}
$$

For $\bar{L}_{G}$ we have:

$$
\begin{aligned}
& \bar{L}_{G}\left(\sigma^{r}, \phi^{r}\right)= \\
& \quad-\frac{1}{2} \sum_{a, b=1}^{9}\left(g_{a} \sigma_{a}^{r}-\mu_{a}+\bar{\mu}_{a}^{0}\right)\left(G^{(-)}\right)_{a b}^{-1}\left(g_{b} \sigma_{b}^{r}-\mu_{b}+\bar{\mu}_{b}^{0}\right) \\
& \quad-\frac{Z}{2} \sum_{a, b=1}^{9} g_{a} \phi_{a}^{r}\left(G^{(+)}\right)_{a b}^{-1} g_{b} \phi_{b}^{r} .
\end{aligned}
$$

2 Despite that the scalar fields are of the main interest in this paper, we still need pseudoscalar fields to fix the model parameters.
Here we introduced Yukawa coupling constants $g_{a}$ :

$$
\begin{gathered}
\sigma_{a}^{\prime}=g_{a} \sigma_{a}^{r}, \quad \phi_{a}=\sqrt{Z} g_{a} \phi_{a}^{r}, \\
g_{1}^{2}=g_{2}^{2}=g_{3}^{2}=g_{8}^{2}=g_{u}^{2}=\left[4 I_{2}^{\Lambda}\left(m_{u}\right)\right]^{-1}, \\
g_{4}^{2}=g_{5}^{2}=g_{6}^{2}=g_{7}^{2}=\left[4 I_{2}^{\Lambda}\left(m_{u}, m_{s}\right)\right]^{-1}, \\
g_{9}^{2}=g_{s}^{2}=\left[4 I_{2}^{\Lambda}\left(m_{s}\right)\right]^{-1}, \\
I_{2}^{\Lambda}\left(m_{u}, m_{s}\right)=\frac{N_{c}}{(2 \pi)^{4}} \int d_{e}^{4} k \frac{\theta\left(\Lambda^{2}-k^{2}\right)}{\left(k^{2}+m_{u}^{2}\right)\left(k^{2}+m_{s}^{2}\right)}= \\
=\frac{3}{(4 \pi)^{2}\left(m_{s}^{2}-m_{u}^{2}\right)}\left[m_{s}^{2} \ln \left(\frac{\Lambda^{2}}{m_{s}^{2}}+1\right)\right. \\
\left.-m_{u}^{2} \ln \left(\frac{\Lambda^{2}}{m_{u}^{2}}+1\right)\right], \\
Z=\left(1-\frac{6 m_{u}}{M_{A_{1}}^{2}}\right)^{-1} \approx 1.44,
\end{gathered}
$$

where we have taken into account $\pi$ - $A_{1}$-transitions leading to an additional $Z$ factor, with $M_{A_{1}}$ being the mass of axial-vector meson (see 21). The renormalized scalar and pseudoscalar fields in 21) are marked with the superscript $r$.

The mass formulae for isovectors and isodublets follow immediately from (18). One just has to look up for the coefficients at $\sigma^{r 2}$ and $\phi^{r 2}$. There are still nondiagonal terms in (20) in the isoscalar sector. This problem is solved by choosing the proper mixing angles both for the scalars and pseudoscalars (see e.g. 34 ). As we are going to introduce the glueball field, the mixing with scalar isoscalar quarkonia will change the situation. One has to consider the mixing among three states, which cannot be described by a single angle. For simplicity, in our estimations we resort to a numerical diagonalization procedure, not to the algebraic one. Concerning the pseudoscalar sector, one can avail oneself with the results given in 34]. All what concerns dealing with the glueball is discussed in the next Section.

\section{Nambu-Jona-Lasinio model with dilaton.}

As we have already mentioned before, we introduce the glueball into our effective Lagrangian, obtained in the previous Section, as a dilaton. For this purpose we use the following principle. Insofar as the QCD Lagrangian, in the chiral limit, is scale invariant, we suppose that our effective meson Lagrangian, motivated by QCD, has also to be scale invariant both before and after SBCS in the case when the current quark masses are equal to zero. Note that the scale anomaly of QCD is reproduced by the dilaton potential. As a result, we come to the following prescription: the dimensional model parameters $G, \Lambda$, and $K$ are replaced by the following rule $G \rightarrow G\left(\chi_{c} / \chi\right)^{2}$, $K \rightarrow K\left(\chi_{c} / \chi\right)^{5}, \Lambda \rightarrow \Lambda\left(\chi / \chi_{c}\right)^{2}$, where $\chi$ is the dilaton field with the vacuum expectation value $\chi_{c}$. Moreover, the constituent quark masses are replaced by the rule 
$m_{a} \rightarrow m_{a}\left(\chi / \chi_{c}\right)$. Concerning the current quark masses, the are left unscaled. This leads to the following formula

$$
\begin{aligned}
\sigma_{a} & =\sigma_{a}^{\prime}-\left(\mu_{a}-\bar{\mu}_{a}^{0}+\mu_{a}^{0}\right) \frac{\chi}{\chi_{c}}+\mu_{a}^{0} \\
& =\sigma_{a}^{\prime}-\left(\mu_{a}-\bar{\mu}_{a}^{0}\right) \frac{\chi}{\chi_{c}}-\mu_{a}^{0} \frac{\chi^{\prime}}{\chi_{c}}
\end{aligned}
$$

where $\mu_{a}^{0}=0, \quad(a=1, \ldots, 7), \mu_{8}^{0}=m_{u}^{0}, \mu_{9}^{0}=-m_{s}^{0} / \sqrt{2}$, and the definition of $\bar{\mu}^{0}$ is given after $(12)$. The difference $\bar{\mu}_{a}^{0}-\mu_{a}^{0}$ is proportional to the 't Hooft interaction constant $K$ (see (7) and (8)) and has conventional scale behaviour, therefore it should be scaled in the same way as $\mu_{a}$. Note that in the r.h.s of (25) $\chi^{\prime}$ denotes the quantum fluctuations of the dilaton field around its vacuum expectation value $\chi_{c}$.

Finally, we come to the following Lagrangian:

$$
\begin{aligned}
& \overline{\mathcal{L}}\left(\sigma^{r}, \phi^{r}, \chi\right)=\mathcal{L}(\chi)+L_{k i n}\left(\sigma^{r}, \phi^{r}\right)+\bar{L}_{G}\left(\sigma^{r}, \phi^{r}, \chi\right) \\
& \quad+\operatorname{tr}\left[-4 m g I_{1}^{\Lambda}(m) \sigma^{r}\left(\frac{\chi}{\chi_{c}}\right)^{3}\right. \\
& \quad+2 g^{2} I_{1}^{\Lambda}(m)\left(\sigma^{r 2}+\phi^{r 2}\right)\left(\frac{\chi}{\chi_{c}}\right)^{2}-m^{2} g^{2} \sigma^{r 2}\left(\frac{\chi}{\chi_{c}}\right)^{2} \\
& \left.\quad+m g \frac{\chi}{\chi_{c}} \sigma^{r}\left(\sigma^{r 2}+\phi^{r 2}\right)-\frac{g^{2}}{4}\left(\sigma^{r 2}+\phi^{r 2}\right)^{2}\right]
\end{aligned}
$$

Here $\mathcal{L}(\chi)$ is the pure dilaton Lagrangian

$$
\mathcal{L}(\chi)=\frac{1}{2}\left(\partial_{\nu} \chi\right)^{2}-V(\chi)
$$

with the potential

$$
V(\chi)=B\left(\frac{\chi}{\chi_{0}}\right)^{4}\left[\ln \left(\frac{\chi}{\chi_{0}}\right)^{4}-1\right]
$$

that has a minimum at $\chi=\chi_{0}$, and the parameter $B$ represents the vacuum energy, when there are no quarks. The curvature of the potential at its minimum determines the bare glueball mass

$$
m_{g}=\frac{4 \sqrt{B}}{\chi_{0}}
$$

The part $L_{k i n}\left(\sigma^{r}, \phi^{r}\right)$ of Lagrangian 26) contains pure kinetic terms

$$
L_{k i n}\left(\sigma^{r}, \phi^{r}\right)=\frac{1}{2}\left(\partial_{\nu} \sigma_{a}^{r}\right)^{2}+\frac{1}{2}\left(\partial_{\nu} \phi_{a}^{r}\right)^{2}
$$

to which we pay no further attention. The next term reads

$$
\begin{aligned}
& \bar{L}_{G}\left(\sigma^{r}, \phi^{r}, \chi\right)= \\
& -\frac{1}{2}\left(\frac{\chi}{\chi_{c}}\right)^{2} \sum_{a, b=1}^{9}\left(g_{a} \sigma_{a}^{r}-\left(\mu_{a}-\bar{\mu}_{a}^{0}+\mu_{a}^{0}\right) \frac{\chi}{\chi_{c}}+\mu_{a}^{0}\right)\left(G^{(-)}\right)_{a b}^{-1} \\
& \times\left(g_{b} \sigma_{b}^{r}-\left(\mu_{b}-\bar{\mu}_{b}^{0}+\mu_{b}^{0}\right) \frac{\chi}{\chi_{c}}+\mu_{b}^{0}\right)
\end{aligned}
$$

$$
\begin{aligned}
& -\frac{Z}{2}\left(\frac{\chi}{\chi_{c}}\right)^{2} \sum_{a, b=1}^{9} g_{a} \phi_{a}^{r}\left(G^{(+)}\right)_{a b}^{-1} g_{b} \phi_{b}^{r}= \\
& -\frac{1}{2}\left(\frac{\chi}{\chi_{c}}\right)^{2} \sum_{a, b=1}^{9}\left(g_{a} \sigma_{a}^{r}-\left(\mu_{a}-\bar{\mu}_{a}^{0}\right) \frac{\chi}{\chi_{c}}-\mu_{a}^{0} \frac{\chi^{\prime}}{\chi_{c}}\right)\left(G^{(-)}\right)_{a b}^{-1} \\
& \times\left(g_{b} \sigma_{b}^{r}-\left(\mu_{b}-\bar{\mu}_{b}^{0}\right) \frac{\chi}{\chi_{c}}-\mu_{b}^{0} \frac{\chi^{\prime}}{\chi_{c}}\right) \\
& -\frac{Z}{2}\left(\frac{\chi}{\chi_{c}}\right)^{2} \sum_{a, b=1}^{9} g_{a} \phi_{a}^{r}\left(G^{(+)}\right)_{a b}^{-1} g_{b} \phi_{b}^{r} .
\end{aligned}
$$

The dilaton field is here expanded around its vacuum expectation value: $\chi=\chi^{\prime}+\chi_{c},\langle\chi\rangle_{0}=\chi_{c},\left\langle\chi^{\prime}\right\rangle_{0}=0$.

Recall that the terms proportional to $m^{0}$ break explicitly chiral and scale invariance in the same way as the current mass term of QCD Lagrangian. Notice also that for our linear $\sigma$-model (26), together with the gap equation (see (41)), lead to a scale-invariant pion term $\sim M_{\pi}^{2} \pi^{2}\left(\chi / \chi_{c}\right)^{2} / 2$ instead of the scale-violating term $\sim M_{\pi}^{2} \pi^{2}\left(\chi / \chi_{c}\right) / 2$ arising in nonlinear $\sigma$-models 33, 38.

As one can see, expanding (31) in power series of $\chi$, we can extract a term that is of order $\chi^{4}$. It can be absorbed by the term in the pure dilaton potential which has the same degree of $\chi$. Obviously, this leads only to a redefinition of the constants $B$ and $\chi_{0}$, which anyway are not known from the very beginning. Moreover, saying in advance, terms like $\chi^{4}$ do not contribute to the divergence of the dilatation current (1) because of their scale invariance.

Let us now consider the vacuum expectation value of the divergence of the dilatation current calculated from the potential of the effective meson-dilaton Lagrangian

$$
\begin{aligned}
& \left\langle\partial_{\mu} S^{\mu}\right\rangle=\left.\left(\sum_{a=8}^{9} \sigma_{a}^{r} \frac{\partial V}{\partial \sigma_{a}^{r}}+\chi \frac{\partial V}{\partial \chi}-4 V\right)\right|_{\substack{\sigma_{a}^{r}=\overline{0} \\
\chi_{c}}} \\
& =4 B\left(\frac{\chi_{c}}{\chi_{0}}\right)^{4}-\sum_{q=u, d, s} m_{q}^{0}\langle\bar{q} q\rangle .
\end{aligned}
$$

Here $V=V(\chi)+\bar{V}\left(\sigma^{r}, \phi^{r}, \chi\right)$, where $\bar{V}\left(\sigma^{r}, \phi^{r}, \chi\right)$ is the potential part of Lagrangian $\overline{\mathcal{L}}\left(\sigma^{r}, \phi^{r}, \chi\right)$. Note that we have simplified (32), taking into account that the quark condensates are related to integrals $I_{1}\left(m_{u}\right)$ and $I_{1}\left(m_{s}\right)$ as follows

$$
4 m_{q} I_{1}^{\Lambda}\left(m_{q}\right)=-\langle\bar{q} q\rangle_{0}, \quad(q=u, d, s),
$$

and that these integrals are connected with constants $G_{a b}^{(-)}$ through gap equations, as it will be shown in the next Section. Comparing the QCD expression (11) with (32), one can see that the quark condensates enter into both formulae in the same way. Equating the right hand sides of (11) and (32),

$$
\mathcal{C}_{g}-\sum_{q=u, d, s} m_{q}^{0}\langle\bar{q} q\rangle=4 B\left(\frac{\chi_{c}}{\chi_{0}}\right)^{4}-\sum_{q=u, d, s} m_{q}^{0}\langle\bar{q} q\rangle
$$


we obtain the correspondence

$$
\mathcal{C}_{g}=4 B\left(\frac{\chi_{c}}{\chi_{0}}\right)^{4}
$$

This equation relates the gluon condensate, whose value we take from other models (see e.g. [39]), to the model parameter $B$. The next step is to investigate the gap equations.

\section{Gap equations}

As usual, gap equations are follow from the requirement that the terms linear in $\sigma^{r}$ and $\chi^{\prime}$ should be absent in our Lagrangian

$$
\begin{aligned}
& \left.\frac{\delta \overline{\mathcal{L}}}{\delta \sigma_{8}^{r}}\right|_{\left(\phi^{r}, \sigma^{r}, \chi^{\prime}\right)=0}=\left.\frac{\delta \overline{\mathcal{L}}}{\delta \sigma_{9}^{r}}\right|_{\left(\phi^{r}, \sigma^{r}, \chi^{\prime}\right)=0} \\
& =\left.\frac{\delta \overline{\mathcal{L}}}{\delta \chi}\right|_{\left(\phi^{r}, \sigma^{r}, \chi^{\prime}\right)=0}=0 .
\end{aligned}
$$

This leads to the following equations

$$
\begin{gathered}
\left(m_{u}-\bar{m}_{u}^{0}\right)\left(G^{(-)}\right)_{88}^{-1}-\frac{m_{s}-\bar{m}_{s}^{0}}{\sqrt{2}}\left(G^{(-)}\right)_{89}^{-1} \\
-8 m_{u} I_{1}^{\Lambda}\left(m_{u}\right)=0 \\
\left(m_{s}-\bar{m}_{s}^{0}\right)\left(G^{(-)}\right)_{99}^{-1}-\sqrt{2}\left(m_{u}-\bar{m}_{u}^{0}\right)\left(G^{(-)}\right)_{98}^{-1} \\
-8 m_{s} I_{1}^{\Lambda}\left(m_{s}\right)=0 \\
4 B\left(\frac{\chi_{c}}{\chi_{0}}\right)^{3} \frac{1}{\chi_{0}} \ln \left(\frac{\chi_{c}}{\chi_{0}}\right)^{4}+\frac{2 A}{\chi_{c}}=0
\end{gathered}
$$

Here

$$
A=\frac{1}{2} \sum_{a, b=1}^{9}\left(\mu_{a}-\bar{\mu}_{a}^{0}\right)\left(G^{(-)}\right)_{a b}^{-1} \mu_{b}^{0}
$$

is proportional to the current quark masses $\mu_{b}^{0} \sim m_{b}^{0}$ and thereby small.

Using (7) and (8), one can rewrite the gap equations (37) and (38) in a well-known form 37

$$
\begin{aligned}
m_{u}^{0}= & m_{u}-8 G m_{u} I_{1}^{\Lambda}\left(m_{u}\right) \\
& -32 K m_{u} m_{s} I_{1}^{\Lambda}\left(m_{u}\right) I_{1}^{\Lambda}\left(m_{s}\right), \\
m_{s}^{0}= & m_{s}-8 G m_{s} I_{1}^{\Lambda}\left(m_{s}\right) \\
& -32 K\left(m_{u} I_{1}^{\Lambda}\left(m_{u}\right)\right)^{2} .
\end{aligned}
$$

The equations discussed above allow us to relate the current quark masses to the rest of model parameters and also to relate the constants $B$ and $\chi_{0}$ to the gluon condensate and $\chi_{c}$. The constituent quark masses, ultraviolet cutoff, and four-quark coupling constants will be fixed, as usual in NJL, by means of the Goldberger-Treimann relation, the $\rho \rightarrow \pi \pi$ decay constant, pion weak decay constant and the mass spectrum of pseudoscalars (For details see [34] and Refs. therein). In the next Section we define $\chi_{c}$, using the bare glueball mass (without mixing effects) as a parameter.
Table 1. The masses of physical the scalar meson states $\sigma_{I}$, $\sigma_{I I}, \sigma_{I I I}$ and the values of the parameters $\chi_{c}, \chi_{0}$, bag constant $B$, and (bare) glueball mass $m_{g}$ (in $\mathrm{MeV}$ ) for two cases: 1 ) $M_{\sigma_{I I I}}=1500 \mathrm{MeV}$ and 2) $M_{\sigma_{I I I}}=1710 \mathrm{MeV}$.

\begin{tabular}{||c|c|c|c|c|c|c|c||}
\hline & $\sigma_{I}$ & $\sigma_{I I}$ & $\sigma_{I I I}$ & $\chi_{c}$ & $\chi_{0}$ & $B,\left[\mathrm{GeV}^{4}\right]$ & $m_{g}$ \\
\hline I & 555 & 1075 & 1500 & 191 & 192 & 0.005 & 1480 \\
II & 555 & 1080 & 1710 & 167 & 168 & 0.005 & 1695 \\
\hline
\end{tabular}

\section{Mass formulae and numerical estimations.}

The potential part of Lagrangian (26) which is quadratic in fields $\sigma^{r}$ and $\chi^{\prime}$ and which we denote as $L^{(2)}$ has the form

$$
\begin{aligned}
& L^{(2)}\left(\sigma^{r}, \phi^{r}, \chi^{\prime}\right)= \\
& -\frac{1}{2} g_{8}^{2}\left\{\left[\left(G^{(-)}\right)_{88}^{-1}-8 I_{1}^{\Lambda}\left(m_{u}\right)\right]+4 m_{u}^{2}\right\} \sigma_{8}^{r 2} \\
& -\frac{1}{2} g_{9}^{2}\left\{\left[\left(G^{(-)}\right)_{99}^{-1}-8 I_{1}^{\Lambda}\left(m_{s}\right)\right]+4 m_{s}^{2}\right\} \sigma_{9}^{r 2} \\
& -g_{8} g_{9}\left(G^{(-)}\right)_{89}^{-1} \sigma^{r}{ }_{8} \sigma_{9}^{r}-2\left(\mathcal{C}_{g}-\frac{A}{4}\right)\left(\frac{\chi^{\prime}}{\chi_{c}}\right)^{2} \\
& -\sum_{a, b=8,9} \frac{\mu_{a}^{0}}{\chi_{c}}\left(G^{(-)}\right)_{a b}^{-1} g_{b} \sigma_{b}^{r} \chi^{\prime} .
\end{aligned}
$$

The dilaton and its interaction with quarkonia does not change the model parameters $m_{u}, m_{s}, \Lambda, G$, and $K$ fixed in our earlier paper 34

$$
\begin{aligned}
& m_{u}=280 \mathrm{MeV}, m_{s}=420 \mathrm{MeV}, \Lambda=1.25 \mathrm{GeV}, \\
& G=4.38 \mathrm{GeV}^{-2}, K=11.2 \mathrm{GeV}^{-5} .
\end{aligned}
$$

As it has been already mentioned, after the dilaton field is introduced into our model, there appear three new parameters: $\chi_{0}, \chi_{c}$, and $B$. To determine these parameters, we use the two equations (35) and (39) and the bare (without mixing effects) glueball mass

$$
m_{g}^{2}=\frac{4 \mathcal{C}_{g}-A}{\chi_{c}^{2}}
$$

We adjust it so that, in the output, the mass of the heaviest meson would be $1500 \mathrm{MeV}$ or $1710 \mathrm{MeV}$, and thereby fix $\chi_{c}$. For the gluon condensate, we use the value $(390 \mathrm{MeV})^{4}$ [39]. The result of our fit is presented in Table 1 where we show the spectrum of three physical scalar isoscalar states $\sigma_{I}, \sigma_{I I}$ and $\sigma_{I I I}$. The last one is associated with the glueball. The parameters $\chi_{0}$ and $B$ are fixed by the gluon condensate and constituent quark masses

$$
\begin{aligned}
& \chi_{0}=\chi_{c} \exp \left(\frac{A}{2 \mathcal{C}_{g}}\right), \\
& B=\frac{\mathcal{C}_{g}}{4} \exp \left(-\frac{2 A}{\mathcal{C}_{g}}\right) .
\end{aligned}
$$

The mixing of scalar isoscalar fields is described by the matrix $b$ that connects the nondiagonalized fields $\sigma^{r}=$ $\left(\sigma_{8}^{r}, \sigma_{9}^{r}, \chi^{\prime}\right)$ with the physical ones $\sigma_{p h}=\left(\sigma_{I}, \sigma_{I I}, \sigma_{I I I}\right)$

$$
\sigma^{r}=b \sigma_{p h} .
$$

The matrix elements of $b$ are given in Table 2 . 
Table 2. Elements of the matrix $b$, describing mixing in the scalar isoscalar sector. The upper table refers to the case $\sigma_{I I I} \equiv$ $f_{0}(1500)$, the lower one to the case $\sigma_{I I I} \equiv f_{0}(1710)$

\begin{tabular}{|c|ccc|}
\hline & $\sigma_{I}$ & $\sigma_{I I}$ & $\sigma_{I I I}$ \\
\hline$\sigma_{u}^{r}$ & 0.9804 & 0.1865 & -0.0636 \\
$\sigma_{s}^{r}$ & -0.1963 & 0.9535 & -0.2288 \\
$\chi^{\prime}$ & 0.0180 & 0.2368 & 0.9714 \\
\hline \hline & $\sigma_{I}$ & $\sigma_{I I}$ & $\sigma_{I I I}$ \\
\hline$\sigma_{u}^{r}$ & 0.9804 & 0.1912 & -0.0474 \\
$\sigma_{s}^{r}$ & -0.1965 & 0.9672 & -0.1609 \\
$\chi^{\prime}$ & 0.0151 & 0.1671 & 0.9858 \\
\hline
\end{tabular}

\section{Decay widths}

Once all parameters are fixed, we can estimate the decay widths for the main strong decay modes of scalar mesons: $\sigma_{l} \rightarrow \pi \pi, \sigma_{l} \rightarrow K K, \sigma_{l} \rightarrow \eta \eta, \sigma_{l} \rightarrow \eta \eta^{\prime}$, and $\sigma_{l} \rightarrow 4 \pi$ where $l=I, I I, I I I$.

The amplitudes that describe the decays are relatively simple in our model. The decays of quarkonia were considered in 34. Here we only give numerical estimates for their decay widths, where the mixing with glueball is taken into account (see Table 2). Below, we discuss only those amplitudes that describe glueball decays. The process $\sigma_{I I I} \rightarrow \pi \pi$ is given by the amplitude

$$
A_{\sigma_{I I I} \rightarrow \pi \pi}=A_{\sigma_{I I I} \rightarrow \pi \pi}^{g}+A_{\sigma_{I I I} \rightarrow \pi \pi}^{q}
$$

which has been divided into two parts. The first part represents the contribution from the pure glueball. It is proportional to the square of the pion mass

$$
A_{\sigma_{I I I} \rightarrow \pi \pi}^{g}=-\frac{M_{\pi}^{2}}{\chi_{c}} b_{\chi \sigma_{I I I}}
$$

where $b_{\chi \sigma_{I I I}}$ represents a corresponding element of the $3 \times 3$ mixing matrix for scalar isoscalar states (see Table 2). This contribution is small (since it is proportional to the current quark mass $m_{u}^{0}$ ), and the process is determined by the second part that describes the decay of the quark component of the glueball

$$
A_{\sigma_{I I I} \rightarrow \pi \pi}^{q}=2 g_{u} m_{u} Z b_{\sigma_{u} \sigma_{I I I}}
$$

Despite the smallness of mixing, $\left|b_{\sigma_{u} \sigma_{I I I}}\right| \ll 1$, this term prevails over the pure glueball contribution because $M_{\pi}^{2}$ is noticeably less than $2 g_{u} m_{u} Z \chi_{c} b_{\sigma_{u} \sigma_{I I I}}$. As a result, the decay width of $\sigma_{I I I}$, if is

$$
\Gamma_{\sigma_{I I I} \rightarrow \pi \pi}=4 \mathrm{MeV}
$$

for $\sigma_{I I I} \equiv f_{0}(1500)$, and

$$
\Gamma_{\sigma_{I I I} \rightarrow \pi \pi}=3 \mathrm{MeV}
$$

for $\sigma_{I I I} \equiv f_{0}(1710)$. As one can see this process occurs with a relatively low rate.

In the case of $K \bar{K}$ channels, the contribution of the pure glueball is also proportional to the mass square of the secondary particle, kaon in this case. But it is rather large, compared to the pion case as $m_{s}^{0} \gg m_{u}^{0}$. In the same way, the amplitude can be split into two contributions

$$
A_{\sigma_{I I I} \rightarrow K \bar{K}}=A_{\sigma_{I I I} \rightarrow K \bar{K}}^{g}+A_{\sigma_{I I I} \rightarrow K \bar{K}}^{q}
$$

where the pure glueball decay into $K \bar{K}$ is represented by amplitude

$$
A_{\sigma_{I I I} \rightarrow K \bar{K}}^{g}=-\frac{2 M_{K}^{2}}{\chi_{c}} b_{\chi \sigma_{I I I}} .
$$

Its value is large and comparable with the quark component contribution

$$
A_{\sigma_{I I I} \rightarrow K \bar{K}}^{q}=2 g_{u} m_{u} Z b_{\sigma_{u} \sigma_{I I I}}-2 \sqrt{2} g_{s} m_{s} Z b_{\sigma_{s} \sigma_{I I I}} .
$$

In this case, the contribution from the quark component is provided by both $u(d)$ and $s$ quarks. In the case that $\sigma_{I I I}$ is $f_{0}(1500)$, we have

$$
\Gamma_{\sigma_{I I I} \rightarrow K \bar{K}}=42 \mathrm{MeV},
$$

and in the other case $\left(\sigma_{I I I} \equiv f_{0}(1710)\right)$

$$
\Gamma_{\sigma_{I I I} \rightarrow K \bar{K}}=90 \mathrm{MeV} \text {. }
$$

Strange quarks contribute more and interfere with the pure glueball part, essentially reducing the decay width (by a factor 3 ).

The amplitude of the decay of a glueball into $\eta \eta$ and $\eta \eta^{\prime}$ can also be considered in the same manner. The only complication is the singlet-octet mixing in the pseudoscalar sector. The corresponding amplitudes are

$$
\begin{aligned}
A_{\sigma_{I I I} \rightarrow \eta \eta}= & A_{\sigma_{I I I} \rightarrow \eta \eta}^{g}+A_{\sigma_{I I I} \rightarrow \eta \eta}^{q} \\
A_{\sigma_{I I I} \rightarrow \eta \eta}^{g}= & -\frac{M_{\eta}^{2}}{\chi_{c}} b_{\chi \sigma_{I I I}}, \\
A_{\sigma_{I I I} \rightarrow \eta \eta}^{q}= & 2 g_{u} m_{u} Z b_{\sigma_{u} \sigma_{I I I}} \sin ^{2} \bar{\theta} \\
& -2 \sqrt{2} g_{s} m_{s} Z b_{\sigma_{s} \sigma_{I I I}} \cos ^{2} \bar{\theta},
\end{aligned}
$$

where $\bar{\theta}=\theta-\theta_{0}$, with $\theta$ being the singlet-octet mixing angle in the pseudoscalar channel, $\theta \approx-19^{\circ}$ 34, and $\theta_{0}$ the ideal mixing angle $\tan \theta_{0}=1 / \sqrt{2}$. The decay widths thereby are:

$$
\Gamma_{\sigma_{I I I} \rightarrow \eta \eta}=25 \mathrm{MeV}
$$

for $\sigma_{I I I} \equiv f_{0}(1500)$, and

$$
\Gamma_{\sigma_{I I I} \rightarrow \eta \eta}=42 \mathrm{MeV}
$$

for $\sigma_{I I I} \equiv f_{0}(1710)$.

For the decay of the glueball into $\eta \eta^{\prime}$, we have the following amplitude

$$
\begin{aligned}
A_{\sigma_{I I I} \rightarrow \eta \eta^{\prime}}= & A_{\sigma_{I I I} \rightarrow \eta \eta^{\prime}}^{g}+A_{\sigma_{I I I} \rightarrow \eta \eta^{\prime}}^{q}, \\
A_{\sigma_{I I I} \rightarrow \eta \eta^{\prime}}^{g}= & 0, \\
A_{\sigma_{I I I} \rightarrow \eta \eta^{\prime}}^{q}= & -2 Z \sin 2 \bar{\theta}\left(g_{u} m_{u} b_{\sigma_{u} \sigma_{I I I}}\right. \\
& \left.+\sqrt{2} g_{s} m_{s} b_{\sigma_{s} \sigma_{I I I}}\right) .
\end{aligned}
$$


The amplitude $A_{\sigma_{I I I} \rightarrow \eta \eta^{\prime}}^{g}$ is equal to zero because there is no decay of a bare glueball into $\eta \eta^{\prime}$. This process occurs only due to the mixing between the glueball and scalar isoscalar quarkonia. The decay widths are as follows

$$
\Gamma_{\sigma_{I I I} \rightarrow \eta \eta^{\prime}}=5 \mathrm{MeV}
$$

for $\sigma_{I I I} \equiv f_{0}(1500)$,

$$
\Gamma_{\sigma_{I I I} \rightarrow \eta \eta^{\prime}}=5 \mathrm{MeV}
$$

for $\sigma_{I I I} \equiv f_{0}(1710)$. The estimate for the decay $f_{0}(1500)$ into $\eta \eta^{\prime}$ is just qualitative because the decay is allowed only due to the finite width of the resonance as its mass lies a little bit below the $\eta \eta^{\prime}$ threshold. The calculation is made for the mass of $f_{0}(1500)$ plus its half-width. For $f_{0}(1710)$, we have a more reliable estimation since the mass is large enough for the decay to be possible. One can see that the order of magnitude for this decay is about $5 \mathrm{MeV}$. The estimate for $f_{0}(1500)$ is not in contradiction with it.

The decays into four pions are estimated as decays proceeding throug two channels: one with two intermediate scalar resonances $(\chi \rightarrow \sigma \sigma \rightarrow 4 \pi)$ and one with only one intermediate scalar resonance $(\chi \rightarrow \sigma 2 \pi \rightarrow 4 \pi)$. Here we neglect the mixing of glueball with quarkonia since the mixing effect is small (it is proportional to $\left.m_{u}^{0}\right)$. 1 The amplitude describing the decay into $2 \pi^{+} 2 \pi^{-}$is as follows

$$
\begin{aligned}
A_{\sigma_{I I I} \rightarrow 2 \pi^{+} 2 \pi^{-}}=A_{\sigma_{I I I} \rightarrow \sigma \sigma} \rightarrow 2 \pi^{+} 2 \pi^{-} & +A_{\sigma_{I I I} \rightarrow \sigma 2 \pi \rightarrow 2 \pi^{+}} 2 \pi^{-}, \\
A_{\sigma_{I I I} \rightarrow \sigma \sigma \rightarrow 2 \pi^{+} 2 \pi^{-}}= & -\frac{32 m_{u}^{4} Z M_{\sigma}^{2}}{F_{\pi}^{2} \chi_{c}}\left(\Delta\left(s_{12}\right) \Delta\left(s_{34}\right)\right. \\
& \left.+\Delta\left(s_{14}\right) \Delta\left(s_{23}\right)\right), \\
A_{\sigma_{I I I} \rightarrow \sigma 2 \pi \rightarrow 2 \pi^{+} 2 \pi^{-}}= & -\frac{16 m_{u}^{4} Z}{F_{\pi}^{2} \chi_{c}}\left(\Delta\left(s_{12}\right)+\Delta\left(s_{34}\right)\right. \\
& \left.+\Delta\left(s_{14}\right)+\Delta\left(s_{23}\right)\right),
\end{aligned}
$$

where $F_{\pi}=93 \mathrm{MeV}$ is the pion week decay constant, $M_{\sigma}$ is the mass of the state $\sigma_{I}$. The function $\Delta(s)$ appears due to the resonant structure of the processes

$$
\Delta(s)=\left(s-M_{\sigma}^{2}+i M_{\sigma} \Gamma_{\sigma}\right)^{-1},
$$

where $\Gamma_{\sigma}$ is the decay width of the $\sigma_{I}$ resonance (see below). This function depends on an invariant mass squared $s_{i j}$ defined as follows

$$
s_{i j}=\left(k_{i}+k_{j}\right)^{2}, \quad(i, j=1, \ldots, 4) .
$$

Here $i$ and $j$ enumerate the momenta $k_{i}$ of pions $\pi^{+}\left(k_{1}\right)$, $\pi^{-}\left(k_{2}\right), \pi^{+}\left(k_{3}\right)$, and $\pi^{-}\left(k_{4}\right)$. The amplitude describing the decay into $2 \pi^{0} \pi^{+} \pi^{-}$has the form

$$
\begin{aligned}
& A_{\sigma_{I I I} \rightarrow 2 \pi^{0} \pi^{+} \pi^{-}}= \\
& \quad A_{\sigma_{I I I} \rightarrow \sigma \sigma \rightarrow 2 \pi^{0} \pi^{+} \pi^{-}}+A_{\sigma_{I I I} \rightarrow \sigma 2 \pi \rightarrow 2 \pi^{0} \pi^{+} \pi^{-}},
\end{aligned}
$$

3 Only the lowest scalar isoscalar resonance is taken into account here. The contribution from $f_{0}(980)$ should be noticeably smaller because of a large mass and a narrow width of $f_{0}(980)$.

$$
\begin{aligned}
& A_{\sigma_{I I I} \rightarrow \sigma \sigma \rightarrow 2 \pi^{0} \pi^{+} \pi^{-}}=-\frac{16 m_{u}^{4} Z M_{\sigma}^{2}}{F_{\pi}^{2} \chi_{c}} \Delta\left(s_{12}\right) \Delta\left(s_{34}\right),(75) \\
& A_{\sigma_{I I I} \rightarrow \sigma 2 \pi \rightarrow 2 \pi^{0} \pi^{+} \pi^{-}}=-\frac{16 m_{u}^{4} Z}{F_{\pi}^{2} \chi_{c}}\left(\Delta\left(s_{12}\right)+\Delta\left(s_{34}\right)\right)(.76)
\end{aligned}
$$

Here $k_{1}$ and $k_{2}$ are momenta of the two $\pi^{0}$, and $s_{12}$ is their invariant mass squared. The indices 3 and 4 stand for $\pi^{+}$ and $\pi^{-}$, respectively.

In the case of the decay into $4 \pi^{0}$, we have

$$
\begin{aligned}
A_{\sigma_{I I I} \rightarrow 4 \pi^{0}}= & A_{\sigma_{I I I} \rightarrow \sigma \sigma \rightarrow 4 \pi^{0}}+A_{\sigma_{I I I} \rightarrow \sigma 2 \pi \rightarrow 4 \pi^{0}},(77) \\
A_{\sigma_{I I I} \rightarrow \sigma \sigma \rightarrow 4 \pi^{0}}= & -\frac{16 m_{u}^{4} Z M_{\sigma}^{2}}{F_{\pi}^{2} \chi_{c}}\left(\Delta\left(s_{12}\right) \Delta\left(s_{34}\right)\right. \\
& \left.+\Delta\left(s_{13}\right) \Delta\left(s_{24}\right)+\Delta\left(s_{14}\right) \Delta\left(s_{23}\right)\right),(78) \\
A_{\sigma_{I I I} \rightarrow \sigma 2 \pi \rightarrow 4 \pi^{0}}= & -\frac{16 m_{u}^{4} Z}{F_{\pi}^{2} \chi_{c}}\left(\Delta\left(s_{12}\right)+\Delta\left(s_{13}\right)+\Delta\left(s_{14}\right)\right. \\
& \left.+\Delta\left(s_{23}\right)+\Delta\left(s_{24}\right)+\Delta\left(s_{34}\right)\right) .
\end{aligned}
$$

From our estimation it follows that in the case, where $\sigma_{I I I}$ is identified with $f_{0}(1500)$, we have the total width

$$
\Gamma_{\sigma_{I I I} \rightarrow 4 \pi}=30 \mathrm{MeV}
$$

and in the other case $\left(\sigma_{I I I} \equiv f_{0}(1710)\right)$

$$
\Gamma_{\sigma_{I I I} \rightarrow 4 \pi}=60 \mathrm{MeV} .
$$

Let us present the decay widths of $\sigma_{I}$ and $\sigma_{I I}$. The state $\sigma_{I}$ that we identify with $f_{0}(400-1200)$ decays mostly into a pair of pions, and this process determines the width of $\sigma_{I}$ :

$$
\Gamma_{\sigma_{I} \rightarrow \pi \pi} \approx 760 \mathrm{MeV} .
$$

The state $\sigma_{I I I}$ does not affect it noticeably, since the mixing of the glueball with $u \bar{u}$ is very small. Therefore, the decay rate for both $\sigma_{I I I} \equiv f_{0}(1500)$ and $\sigma_{I I I} \equiv f_{0}(1710)$ is approximately the same in magnitude.

The decay of the state $\sigma_{I I}$ that we identify with $f_{0}(980)$ into pions is determined by the quark component and is slightly reduced by the glueball component because of mixing with the $s \bar{s}$ quarkonium. We obtain

$$
\Gamma_{\sigma_{I I} \rightarrow \pi \pi}=17 \mathrm{MeV}
$$

if $\sigma_{I I I} \equiv f_{0}(1500)$ and

$$
\Gamma_{\sigma_{I I} \rightarrow \pi \pi}=15 \mathrm{MeV}
$$

if $\sigma_{I I I} \equiv f_{0}(1710)$. From experiment, we know that its decay width lies within the interval from $40 \mathrm{MeV}$ to 100 $\mathrm{MeV}$. Concerning the process $\sigma_{I I} \rightarrow \pi \pi$, we obtain a decay width that is lower than the experimental one. Notice that this prediction is completely based on singlet-octet mixing following from the 't Hooft interaction [34] where dilaton effects do play a minor role. The decay into $K \bar{K}$ can also be taken into account. From experiment we learn that the decay into $K \bar{K}$ can contribute about $30 \%$ to the total width [4]. Our estimates for decays of the glueball are collected in Table 3. 
Table 3. The partial and total decay widths (in $\mathrm{MeV}$ ) of the glueball for two cases: $\sigma_{I I I} \equiv f_{0}(1500)$ and $\sigma_{I I I} \equiv f_{0}(1710)$, and experimental values of decay widths of $f_{0}(1500)$ and $f_{0}(1710)$ 幽.

\begin{tabular}{||c|r|r|r|r|r|r|c||}
\hline & $\Gamma_{\pi \pi}$ & $\Gamma_{K \bar{K}}$ & $\Gamma_{\eta \eta}$ & $\Gamma_{\eta \eta^{\prime}}$ & $\Gamma_{4 \pi}$ & $\Gamma_{\text {tot }}$ & $\Gamma_{\text {tot }}^{\text {exp }}$ \\
\hline$f_{0}(1500)$ & 4 & 42 & 25 & 5 & 30 & 100 & 112 \\
$f_{0}(1710)$ & 3 & 90 & 42 & 5 & 60 & 200 & 130 \\
\hline
\end{tabular}

\section{Conclusion}

As it was mentioned in the Introduction, the inclusion of a scalar glueball into the effective meson Lagrangian is quite an ambiguous procedure. The goal of our paper is to find the most physically justified way to do this. In the approach presented above, we assume that (with the exception of the dilaton potential) scale invariance holds for the effective Lagrangian before and after SBCS in the chiral limit. The terms depending on current quark masses break both the chiral and scale invariance, in accordance with QCD. This leads to the requirement that we should introduce the dilaton field into the constituent quark masses while the current quark masses remain unscaled.

In this version of a scaled NJL model, the terms that describe mixing of the glueball with quarkonia are also proportional to current quark masses. The same is true for the amplitudes describing decays of the glueball into pairs of pseudoscalars. Insofar as the masses of current quarks are small in comparison with the other model parameters (constituent quark mass, $\chi_{c}, \Lambda$, and so on), this results in a small mixing of the glueball with quarkonia, relatively small rates for decays of the glueball into $\pi \pi$, and only slightly changes the decay width of $f_{0}(980) \rightarrow \pi \pi$ calculated before introducing the glueball 34 . The decay of the glueball into two pions is mostly determined by its $q \bar{q}$ admixture despite the small mixing. The mixing coefficient here, although being small $\left(\sim-0.06\right.$ if $\sigma_{I I I} \equiv f_{0}(1500)$ ), is multiplied by a relatively large constant describing the decay of the $\sigma$-meson into a pair of pions.

In the case of the $K K$ channel, both the gluonic and quark components play an important role since the interference between the gluonic and quark amplitudes is large. The relatively small contribution from the $u \bar{u}$ component slightly increases the decay rate of the glueball. But the contribution from the $s \bar{s}$ component reduces the contribution from the pure glueball by factor 3 .

The decay into $\eta \eta$ is mostly determined by the glueball component. The mixing of the glueball with $\bar{s} s$ reduces the decay rate but not significantly. The decay into $\eta \eta^{\prime}$ is less than into $\eta \eta$ and is allowed only due to the mixing of quarkonia with the glueball. This process serves as a measure of this mixing. However, in the case of $f_{0}(1500)$, it is difficult to give reliable estimates for its rate because the process occurs near the threshold.

Decays into 4 pions are represented by two processes. In the first one, two intermediate scalar resonances are born by the glueball with their subsequent decay into two pairs of pions. In the second process, only one intermediate scalar resonance together with a pair of pions are produced immediately after the decay of the glueball. Then, the scalar resonance decays into pions. From our calculations it follows that the second process is dominant and two scalar resonances are less probable to appear.

The total width of the third scalar isoscalar state is estimated to be about $100 \mathrm{MeV}$ for $M_{\sigma_{I I I}}=1500 \mathrm{MeV}$ and $200 \mathrm{MeV}$ for $M_{\sigma_{I I I}}=1710 \mathrm{MeV}$. If we assume that the $f_{0}(1500)$ state is the scalar glueball, the total decay width derived from our model is close to the experimental value $(112 \mathrm{MeV})$. Unfortunately, the detailed data on the branching ratios of $f_{0}(1500)$ are not reliable and controversial [4].

In conclusion, we would like to note that, in our model, the width of the decay of a glueball into two pions is small, because the amplitude describing this decay, is proportional to the current mass of $u$-quark $\left(\sim M_{\pi}^{2} \sim m_{u}^{0}\right)$ and does not depend on momenta. The latter in the chiral limit formally disagrees with the low-energy theorems obtained in paper [40]. In general, we could consider a version of our model containing momentum-dependent vertices, whose momentum dependence is in agreement with these low-energy theorems. However, such a momentum dependence of the amplitude leads to too large decay width of a heavy glueball (see 33]), which contradicts the experimental data. This witnesses to the fact that these low-energy theorems are not justified to be applied in the case of a heavy glueball.

The results obtained here correspond to the leading order in $1 / N_{c}$ expansion (Hartree-Fock approximation). Next-to-leading order corrections can to an extent change the final results. Note also that, in the energy region under consideration $(\sim 1500 \mathrm{MeV})$, we work on the brim of the validity of exploiting the chiral symmetry that was used to construct our effective Lagrangian. Thus, we can consider our results as rather qualitative. Nevertheless, a satisfactory agreement with experimental data is obtained for the total width of $f_{0}(1500)$.

We are going to use this approach in our future work for describing both glueballs and ground and radially excited scalar meson nonets which lie it the energy interval from 0.4 to $1.71 \mathrm{GeV}$. Small mixing angles make us hope that introducing the glueball into our model will not change the whole picture dramatically.

We are grateful to Prof. S.B. Gerasimov, Dr. A.E. Dorokhov, and Dr. N.I. Kochelev for useful discussions. The work is supported by RFBR Grant 00-02-17190, the Heisenberg-Landau program 2000 and the Graduiertenkolleg "Elementarteilchenphysik" of the Humboldt University, Berlin.

\section{References}

1. V. De Alfaro et al. Currents in Hadron Physics, American Elsevier Publ. Comp. Inc., New York, 1973.

2. M. K. Volkov, Sov. J. Part. and Nuclei 17, 186 (1986); D. Ebert and H. Reinhardt, Nucl. Phys. B271, 188 (1986); M. K. Volkov, Phys. Part. Nucl. 24, 35 (1993).

3. G.E. Brown and A.D. Jackson, The Nucleon-Nucleon Interaction, North-Holland, Amsterdam, 1976; B.D. Serot 
and J.D. Walecka, Advances in Nuclear Physics, ed. by J.W. Negele and E.Vogt (Plenum, New. York, 1986), vol. 16 .

4. C. Caso et al., Eur. Phys. J. C3, 1 (1998).

5. H. Comm, P. Jain, R. Johnson, and J. Schechter, Phys. Rev. D33, 3476 (1986); U.-G. Meissner and N. Kaiser, Phys. Rev. D35, 2859 (1987); U.-G. Meissner, R. Johnson, N. Park, and J. Schechter, Phys. Rev. D37, 1285 (1988).

6. P. Jain, R. Johnson, and J. Schechter, Phys. Rev. D38, 1571 (1988).

7. J. Ellis, J. Kapusta, and K.A. Olive, Nucl. Phys. B348, 345 (1991); Phys. Lett. B273, 259 (1991).

8. E.K. Heide, S. Rudaz, P. J. Ellis, Phys. Lett. B293, 259 (1992).

9. T. Waindzoch and J. Wambach, Phys. Lett. B295, 16 (1992).

10. G. Ripka and M. Jaminon, Ann. Phys. (N.Y.) 218, 51 (1992); M. Jaminon and G. Rapha, Nucl. Phys. A564, 551 (1993).

11. K.Kusaka and W. Weise, Z. Phys. A343, 229 (1992); Phys. Lett. B288, 6 (1992).

12. K. Tsushima and D. O. Riska, Nucl. Phys. A560, 955 (1993).

13. I. Mishustin, J. Bondorf, and M. Rho, Nucl. Phys. A555, 215 (1993).

14. I.N. Mishustin and W. Greiner, J. Phys. G: Nucl. Part. Phys. 19, L101 (1993).

15. L. S. Celenza, B. Huang, H. Wang, and C. M. Shakin, preprint BCCNT:99/111/283, Brooklin Coll., N.Y., 1999.

16. L.S. Cenenza, Shun-fu Gao, Bo Huangsheng Wang, C.M. Shakin, Phys. Rev. C61, 035201 (2000).

17. M.K.Volkov, V.L.Yudichev, Part. At. Nucl. 31, 576 (2000); M. K. Volkov and V. L. Yudichev, Int. J. Mod. Phys. A14, 4621 (1999); M. K. Volkov and V. L. Yudichev, Phys. At. Nucl. 63(8), 2000 (at press); hep-ph/9905368.

18. C. Amsler et al. Phys. Lett. B353, 425 (1995); C. Amsler and F. Close, Phys. Lett. B353, 385 (1995).

19. S. Narison, Nucl. Phys. B509, 312 (1998).

20. V. V. Anisovich, D. V. Bugg and A. V. Sarantsev, Phys. Rev. D58, 111503 (1998).

21. N.A. Törnqvist, M. Roos, Phys. Rev. Lett. 76, 1575 (1996).

22. J. Cugnon, M. Jaminon and B. Van den Bosche, Nucl. Phys. A598, 515 (1996); M. Jaminon and B. Van den Bosche, Nucl. Phys. A619, 285 (1997).

23. J. Sexton, A. Vaccarino, D. Weingarten, Phys. Rev. Lett. 75, 4563 (1995).

24. W. Lee and D. Weingarten, Phys. Rev. D59, 094508 (1999).

25. A. Vaccarino and D. Weingarten, hep-lat/9910007.

26. A. Palano, Nucl. Phys. Proc. Suppl. 39BC, 287 (1995).

27. Eef van Beveren and G. Rupp, hep-ph/9806246.

28. M. K. Volkov and D. Ebert, Sov. J. Nucl. Phys. 36, 736 (1982); Z. Phys. C16, 205 (1983); M. K. Volkov, Ann. Phys. 157, 282 (1984).

29. M. K. Volkov, C. Weiss, Phys. Rev. D56, 221 (1997).

30. M. K. Volkov, Phys. At. Nucl. 60, 1920 (1997).

31. K. Kusaka, M. K. Volkov and W. Weise, Phys. Lett. B302, 145 (1993).

32. A. A. Andrianov, V. A. Andrianov, V. Yu. Novozhilov and Yu. V. Novozhilov, JETP Lett. 43, 719 (1986); A. A. Andrianov, V. A. Andrianov, D. Ebert and T. Feldmann,
Int. J. Mod. Phys. A12, 5589 (1997); A. A. Andrianov and V. A. Andrianov, Z. Phys. C55, 435 (1992); A. A. Andrianov, V. A. Andrianov, Yu. V. Novozhilov and V. Yu. Novozhilov, Phys. Lett. B186, 401 (1987).

33. J. Ellis and J. Lánik, Phys. Lett. B150, 289 (1984); J. Ellis and J. Lánik, Phys. Lett. B175, 83 (1986); J. Lánik, Acta Phys. Slov. 35, 343 (1985); B.A. Campbell, J.Ellis and K.A. Olive, Nucl. Phys. B345, 57 (1990).

34. M. K. Volkov, M. Nagy and V. L. Yudichev, Nuovo Cim. A112, 225 (1999).

35. G. 't Hooft, Phys. Rev. D14, 3432 (1976); A.E. Dorokhov, N.I. Kochelev, Z.Phys. C37, 377 (1988); A.E. Dorokhov, N.I. Kochelev, Sov. J. Nucl. Phys. 52, 135 (1990); A.E. Dorokhov, N.I. Kochelev, Sov. J. Part. Nucl. 23, 522 (1992).

36. H. Vogl and W. Weise, Progr. Part. Nucl. Phys. 27, 195 (1991).

37. S. P. Klevansky, Rev. Mod. Phys. 64, 649 (1992).

38. N. O. Agasyan, D. Ebert, E.-M. Ilgenfritz, Nucl. Phys. A637, 135 (1998).

39. D. J. Broadhurst et al., Phys. Lett. B329, 103 (1994); B. V. Geshkenbein, Phys. At. Nucl. 58, 1171 (1995); S. Narison, Phys. Lett. B387, 162 (1996).

40. M.A. Shifman, Phys. Rept. 209, 341 (1991). 